\title{
Design of Intelligent Car Based on Single Chip Processor STC89C52
}

\author{
Li Xin ${ }^{1, a}$, Xiang Qiang ${ }^{1, b, 凹}$ \\ ${ }^{1}$ College of Electrical Information, Southwest University for Nationalities, Chengdu 610041, China \\ aemail: 1009132414@qq.com, bemail:xiangqiang@swun.edu.cn
}

Key words: Intelligent tracking car, DC motor, Infrared electric sensor, PWM, Mice Maze

\begin{abstract}
Smart car is a kind of system which can precisely along the path of automatic driving mazes to the end point and return to the starting point accurately according to the optimal path, Under the effect of control system. In this paper, the hardware/software design and implementation of the intelligent tracking car control system are introduced. This tracking car uses three rows of laser sensors to collect road information, software to judge its effectiveness by the corresponding algorithm, control algorithms to control vehicle steering. The proper duty ratio of PWM wave is gived by single chip microcomputer to control the motor speed, and the positive\&negative operation of motor is drived by $\mathrm{H}$ bridge. The smart car can return to the starting point along the optimal path after reach the end point. The smart car can complete the tracking task preferably, and is able to complete the prescribed path with faster speed, always maintains stable operation.
\end{abstract}

\section{Introduction}

The tracking car competition is an activity carried out for promoting higher educational reform, cultivating undergraduates the ability of practice, innovation and team-work spirit. Manufacturing photoelectric tracking car is divided into two parts: hardware manufacturing and software programming. This article proposes a design scheme for photoelectric tracking car. The experiment indicates that this scheme can adjust the speed and angle of car body, combining with all kinds of track's traffic information, in order to ensure cars can finish the full distance in a minimum duration.

\section{Systematic overall hardware structure design and working principle brief introduction}

Intelligent car's overall hardware structure design

The structures of the car mainly divided into a few parts following:

1. Power module. Because cars adopt the battery to supply electricity, reasonable design is a power module, the premise of car's stable operation.

2. Tracking module. It's made up of several phototubes, using for exploring the position of black line, judge if there is black line or not through the change of reflected infrared.

3. driver module of electric motor. As the SCM's current output is limited, and it cannot drive motor to work directly, thus it needs to be driven by appropriative circuit. This article takes $\mathrm{H}$ bridge driver chip L298N that is used commonly for an example.

4.One-chip computer module. The whole design selects 8-bit microcontroller STC89C52 ${ }^{[4]}$ to finish the treatment of collecting data and controling command of each driver, the processing speed of $12 \mathrm{M}$ can deal with all kinds of road information in time and control the direction of cars, and the power dissipation is low, the collocation is simple, and the price is comparatively cheap.

Intelligent tracking car hardware structure diagram as shown in figure 1 [2] : 


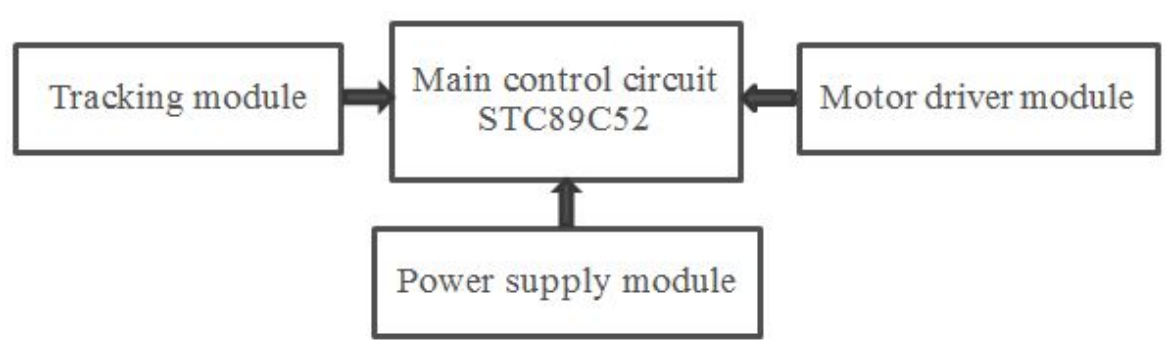

Fig.1 intelligent tracking car hardware structure diagram

Introduction of working principle of the intelligent car

In this design, tracking refers to the car in the white floor walking along the black line $2.5 \mathrm{~cm}$ wide. The tube of infared sensor emits infared, which received by the receiving tube. Simultaneously, the receiving quantity is converted into electrical signals, after processing, signal amplification circuit by MCU to port P1, after processing, the signal by the port output to the motor drive circuit L298N chip, so as to achieve the purpose of driving the car to walk and tracking.

\section{Hardware system design of intelligent tracking the car}

Power supply module

The electricity of power supply module is supplied by two ways, and we can use one way to supply power for single chip microcomputer, lamp etc. and the other way provide the working voltage of L298N, photocell, and motor. Besides, the drive voltage of L298N is given directly by the battery without any treatment.

\section{Profile of master control module STC8952}

TC89C52 chips $40+5$ v power supply pins, 20 pins grounded.XTAL1 of 18 pin and XTAL2 of 19 pin connect crystal which vibration frequency is $12 \mathrm{MHZ}$, and connect $30 \mathrm{pf}$ capacitors.9 pin RST connect reset circuit outside, providing electric reset for single chip microcomputer. Pin 10 and 11 pins of P3 mouth as the communication terminal of single chip microcomputer ,namely, serial input port and serial output needed to complete the program download. The eight ports of the output of sensor send signals correspondingly into microcontroller through the P1 port. After processing , P00 P05 output to EN1, EN2, IN1 IN4 pin of L2938N, finishing controlling the car.

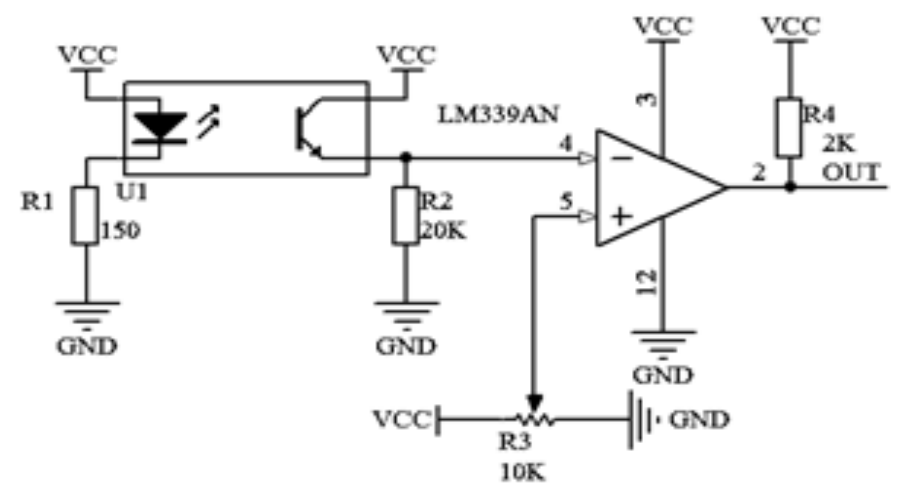

Fig.2 Loop circuit diagram

The design of tracking module

This design is adopted eight ST188 models of infrared electric sensor. The parts of photocell distribution adopt the 332 layout. This method can distinguish every port accurately and simply, control algorithm of program is simple and the car is controlled stably.

ST188 type infrared electric sensor consists of a high power infrared photodiode and a highly 
sensitive receiving infrared photoelectric triode (NPN). When it is used to compare two voltages, an arbitrary input end plus a fixed voltage as reference voltage, and the other end with a voltage signals to be compared. When the voltage of "+" terminal is higher than "-" side, the output pipe is cut off, which means the output side disconnecting. When the voltage of "-" above "+" side, the output tube saturated, equivalent to the output side connects a low potential. The difference between the two input voltages is more than $10 \mathrm{mv}$ which can reliably ensure that the output from one state to another state. Therefore, the LM339 used in weak signal detection is ideal [5].Loop circuit diagram as shown in figure 2 .

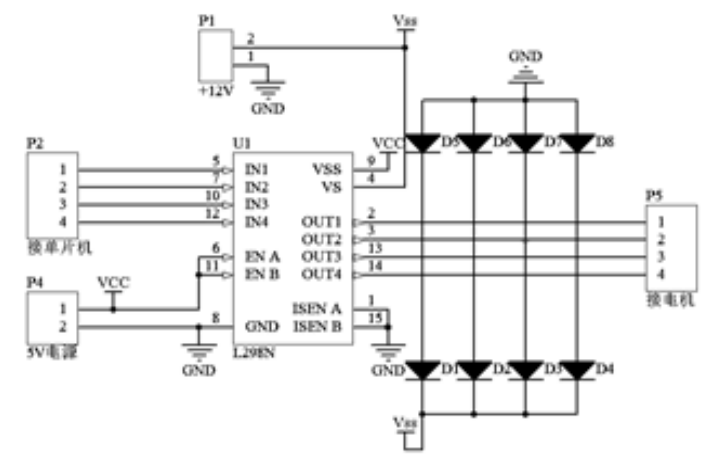

Fig.3 Motor driver module

Motor driver module

This module selects the L298N chip to control. There are three signals and 6 pins to control each electrical machine. And the six pins is linked with P0.0 P0.5 of STC89C52 single-chip microcomputer respectively. Here the working voltage of the chip is $+5 \mathrm{v}$. This design adopts the way of two kinds of outputting to control a motor. Namely the OUT1 and OUT2 control motor 1, OUT3 and OUT4 control motor 2.In figure 5, D1 D8 is clamp diode .Motor, at the instant of stopping, will produce a reverse current, and after cutting the magnetic field can produce a reverse electromotive force. The goal to set these clamp diodes is to prevent the counter electromotive force from burning out the chip, so as to protect them.

Motor driver module as shown in figure 3[6] : 


\section{Software design of intelligent tracking car}

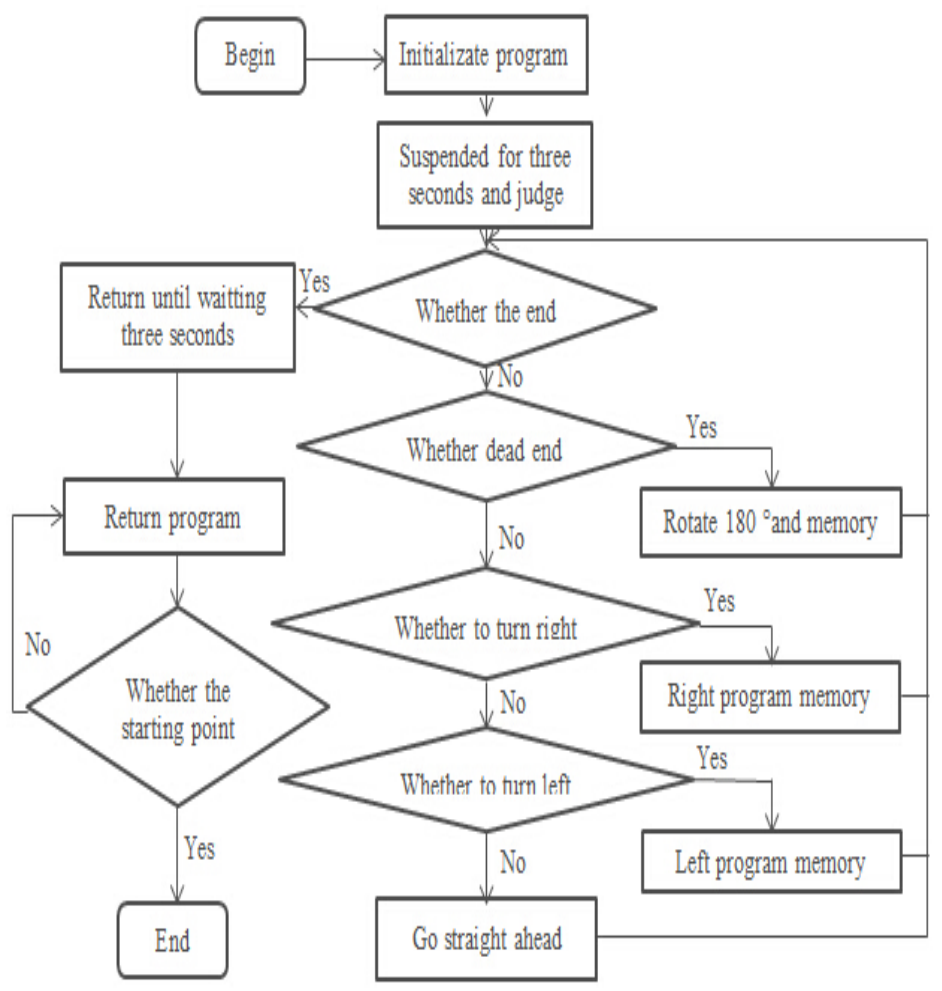

Fig.4 program flow chart

The key point of MCU to complete controlling the automatic car is performing the corresponding software to achieve .In this design, the structured design method is adopted and $\mathrm{C}$ language is used to realize the corresponding functions. Detailed flow chart as shown in figure 4.

The program includes the main program module which is responsible for calling various of subprograms; The initialization module is responsible for initializing all parts; the delay module for the software time delay; PWM module for motor speed adjustment ; The run module for the straight line, the right or left defection and turn, $180^{\circ}$ turn and the stop at the terminal; Memory detection module of tracing for judging each intersection status and recording it; Tracking return module for $t$ calling recorded data and along the optimal path to return

The illustration of several main function modules is showed below.

Tracking memory testing module

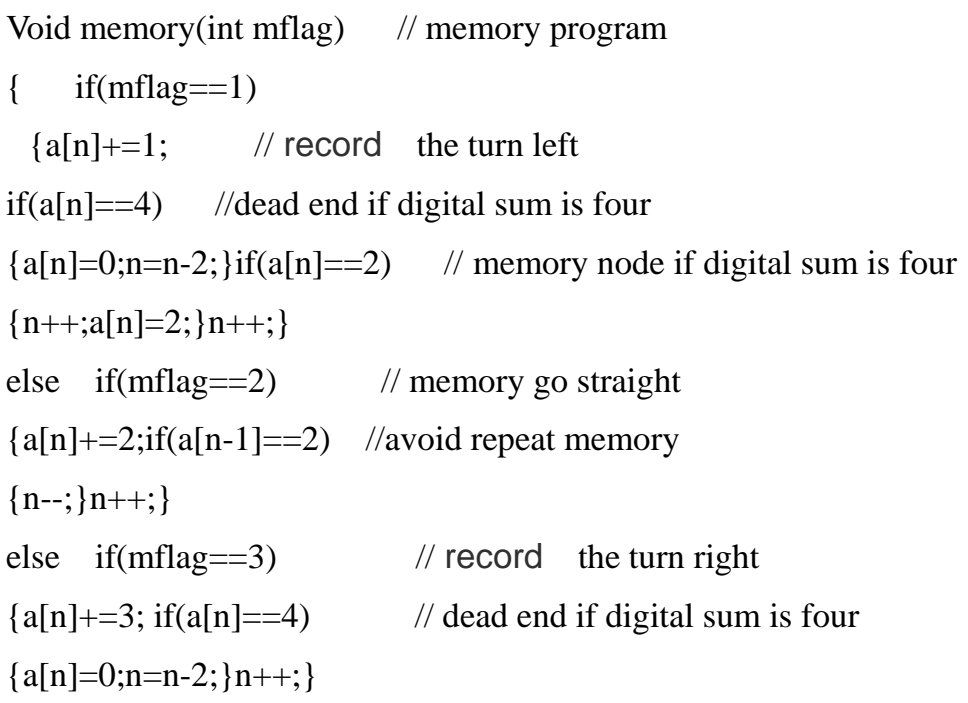




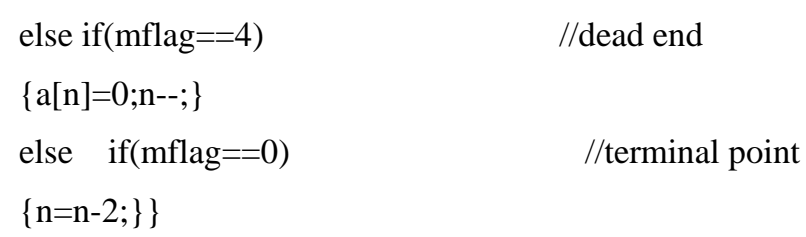

The memory program adopts the left priority principle, so when meet the dead end it needs to consider the problems of the overlapping route and memorizing crossing. The memory program ingeniously solves those problems with algorithms. And the go straight program may be adopted more than once when the car goes straight, the judge in the program can avoid to repeat memory.

Tracking Back To the Module

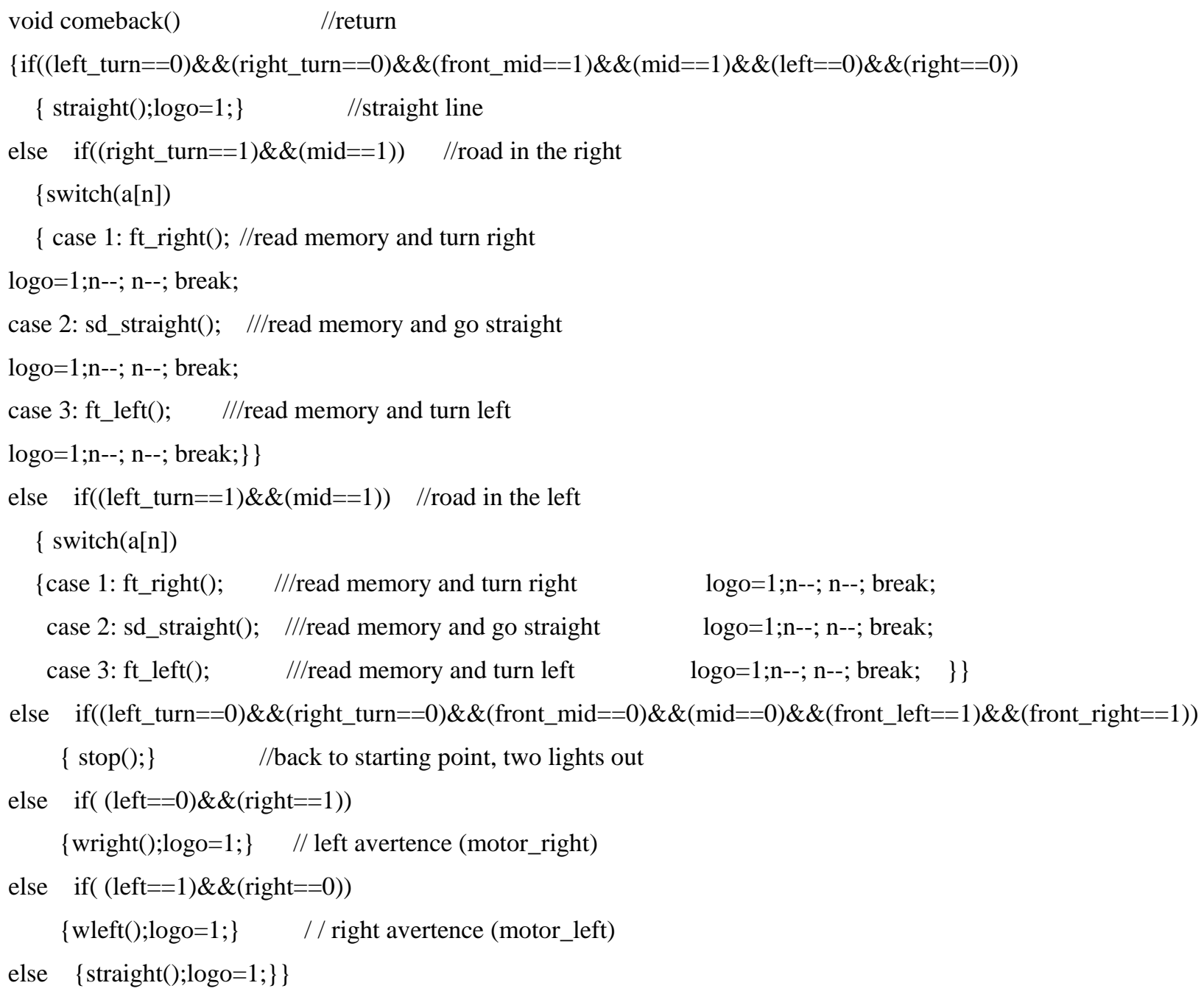

In return module, the corresponding tracing module with the left priority principle replaces by the right priority principle. Since the memory module stores the route and the turning point, once read the crossing, the program should ignore the go straight memory. The program finishes the crossing memory by the use of memory jumping algorithm. Finally, the car returns to the starting point.

\section{Conclusions}

The construction of hardware circuit and the design of software are introduced in this paper. The system uses STC89C5 as CPU to control and finish the route according the established thinking. The optimal route algorithm was proposed after the tracing algorithm, improving the intelligence of the car greatly, consequently, realizing the intelligent tracing movement on the fixed track. Through practical experiment, the system can make the car find route automatically and return precisely. In general, the system is relatively simple, low cost and easy to achieve. 


\section{Reference}

[1]. Liu Yuanming, Li Daolin, Han Xupeng. Induction of tracking car [j]. The design and implementation of electronic design engineering. 2011.19 (10) : $70 \sim 73$.

[2]. Zhu Tao. STC89C52 microcontroller based intelligent tracking car design [j]. Computer knowledge and technology. 2011, 31:7751-7753 + 7758 .

[3]. Dong Leigang Cui Xiaowei, Zhang Dan. The design of intelligent tracking electric car [j]. Journal of daqing normal university journal. 2010.30 (6) $: 8$ 10

[4]. Zhang Yigang, Peng Yu, Zhao Guangquan. Single chip microcomputer principle and interface technology [m]. Beijing: people's posts and telecommunications publishing house, 2011

[5]. Wu Jianping, Yin Zhanguo . Reflecting type of infrared sensor in the application of autonomous navigation car [J]. China measurement technology, 2004 (6) : 21-23.

[6]. Gao Yuehua. Based on the infrared electric sensor of automatic tracking system of smart car design [J]. Journal of photoelectric technology, 2009 (2) : 1-5 